\title{
Gravidez na adolescência e exclusão social: análise de disparidades intra-urbanas
}

\author{
Cristina Maria Duarte, ${ }^{1}$ Vânia Barbosa do Nascimento ${ }^{2}$ \\ e Marco Akerman ${ }^{1}$
}

Como citar Duarte CM, Nascimento VB, Akerman M. Gravidez na adolescência e exclusão social: análise de disparidades intra-urbanas. Rev Panam Salud Publica. 2006;19(4):236-43.

RESUMO Objetivo. Comparar as adolescentes que residiam em quatro áreas com diferentes graus de exclusão social no Município de Santo André, Estado de São Paulo, em relação ao nível de escolaridade, o peso ao nascer e a idade gestacional dos bebês e as taxas de fecundidade específicas de cada área no ano de 1998.

Método. Foi realizado um estudo transversal ecológico com 1314 adolescentes. As quatro áreas utilizadas foram as previamente identificadas no Mapa da exclusão/inclusão social da cidade de Santo André, sendo a área 1 a de exclusão social mais pronunciada e a 4 a de menor exclusão social. Os dados das adolescentes e de seus recém-nascidos foram coletados através do Sistema de Informação sobre Nascidos Vivos (SINASC), e as informações socioeconômicas do Município de Santo André foram obtidas da Fundação Sistema Estadual de Análise de Dados (SEADE), do Instituto Brasileiro de Geografia e Estatística (IBGE) e do Mapa da exclusão/ inclusão social da cidade de Santo André.

Resultados. O nível de escolaridade mostrou uma relação estatisticamente significativa com as áreas mais pobres, que concentravam o maior número de adolescentes com menos escolaridade. Quanto à distribuição do baixo peso ao nascer, 76,8\% dos bebês nascidos com < $2500 \mathrm{~g}$ encontravam-se nas áreas mais pobres da cidade. A maior taxa de fecundidade (35,7 em 1000 adolescentes) também esteve associada às piores condições socioeconômicas, enquanto que a menor taxa $(12,1 \mathrm{em} 1000)$ foi observada na área mais favorecida. A freqüência de bebês prematuros não foi diferente entre as quatro áreas $(\mathrm{P}=0,81)$.

Conclusões. Os resultados mostram que mais adolescentes de baixa escolaridade e menor nível socioeconômico tiveram mais filhos. É necessário promover ações específicas para evitar a gravidez nesse grupo e para incentivar a inclusão social dessas adolescentes e de seus filhos, abrindo a eles perspectivas de modificar a sua condição.

Palavras-chave Classe social, distribuição espacial, gravidez na adolescência, Brasil.

1 Faculdade de Medicina do ABC, Departamento de Saúde da Coletividade, Disciplina de Saúde Coletiva, Santo André (SP), Brasil. Enviar correspondência para Marco Akerman no seguinte endereço: Rua Dona Brígida 232, casa 32, CEP 04111080, São Paulo, SP, Brasil. E-mail: akermanm.ops@ terra.com.br

Secretaria Municipal de Saúde de Santo André e Faculdade de Medicina do ABC, Departamento de Saúde da Coletividade, Disciplina de Saúde Coletiva.
A saúde sexual e reprodutiva da adolescente tem gerado muita preocupação nas organizações de saúde nacionais e internacionais, pelas suas repercussões físicas, psicológicas e sociais $(1,2)$. Atualmente, cerca de $10 \%$ do total de nascimentos mundiais anuais são de mães adolescentes- cerca de 14 milhões de nascimentos por ano (3). Em 1999, dados brasileiros mostraram cerca de 700000 partos entre adolescentes, sendo $32000 \mathrm{em}$ menores de 14 anos (4).

É importante salientar que esse não é um problema tipicamente brasileiro, sendo observado em todo o mundo. 
Além disso, existem diferenças tanto entre países como entre regiões de um mesmo país, determinadas pelo nível de desenvolvimento nacional, que, por sua vez, é reflexo das condições econômicas, das diferenças culturais e do acesso aos serviços de saúde e aos métodos contraceptivos (5).

A maioria dos estudos sobre o tema enfoca aspectos orgânicos, comportamentais e educacionais das adolescentes, mas carece de contextualização mais ampla, pois, apesar do vasto conhecimento produzido nos últimos anos sobre a saúde das adolescentes, o impacto desse conhecimento na prevenção da gravidez não planejada ainda é baixo (6). Nesse debate, a inserção de novas dimensões de análise, como a categoria territorial, pode contribuir para uma melhor compreensão do fenômeno da gravidez na adolescência.

Alguns autores (7-10) estudaram a distribuição de agravos à saúde utilizando técnicas e indicadores alternativos e observaram que existem desigualdades em saúde entre bairros de uma mesma cidade. Eles concluíram que as populações não se distribuem por acaso nas unidades territoriais de uma cidade; tendem a formar conglomerados humanos que compartilham características relativamente similares de natureza social e econômica. A partir disso, seria necessário que se formulassem políticas públicas específicas para as condições de vida e saúde de cada grupo $(9,11)$. Portanto, é preciso que a análise de dados epidemiológicos seja desagregada entre territórios de uma mesma cidade, para que essas diferenças possam ser consideradas na formulação de políticas compatíveis com a diversidade intra-urbana (12).

A exclusão social é a impossibilidade de partilhar os benefícios produzidos pela sociedade. Leva à vivência da privação, da recusa, do abandono e da expulsão. Não se trata de um processo individual, embora atinja pessoas, mas de uma lógica que está presente nas várias formas de relações econômicas, sociais, culturais e políticas. Essa situação de privação coletiva é o que se entende por exclusão social (13).

$\mathrm{O}$ governo do Reino Unido argumenta que a exclusão social aumenta o risco da gravidez na adolescência e que os fatores educacionais são uma das dimensões dessa exclusão $(14,15)$. Bonnell et al. (16) testaram essa hipótese com estudantes entre 13 e 14 anos e encontraram maior risco para gravidez na adolescência entre as estudantes que não gostavam da escola.

Garrafa (17) afirma que é através do reconhecimento das diferenças e das necessidades diversas dos sujeitos sociais que se pode alcançar a igualdade. Isso remete ao conceito de eqüidade, que seria a disposição de reconhecer igualmente o direito de cada um a partir de suas diferenças.

O objetivo deste artigo foi comparar as adolescentes que residiam em quatro áreas com diferentes graus de exclusão social no Município de Santo André, Estado de São Paulo, Brasil, em relação ao nível de escolaridade, o peso ao nascer e a idade gestacional dos bebês e as taxas de fecundidade específicas de cada área no ano de 1998.

\section{MATERIAIS E MÉTODOS}

O Município de Santo André pertence à Grande São Paulo, juntamente com outros 39 municípios, e faz fronteira com 10 deles. Em 1998, a população total era de 629502 habitantes, sendo 58912 adolescentes do sexo feminino (10 a 19 anos) (18), o que correspondia a $9,4 \%$ da população do Município. O número total de nascidos vivos no ano foi de 11043 , e de natimortos, 127. A taxa de mortalidade geral foi de 6,6 por 1000 habitantes, o coeficiente de mortalidade infantil de 17,6 por 1000 vivos nascidos e o coeficiente de mortalidade materna de 52,7 por 100000 nascidos vivos (19).

Foi realizado um estudo transversal ecológico com adolescentes que tiveram filhos e residiam no Município de Santo André no período de $1^{\circ}$ de janeiro a 31 de dezembro de 1998 . Foram consideradas residentes as moradoras de bairros pertencentes às áreas censitárias oficiais quando do nascimento de seus filhos $(1,20)$.

$\mathrm{O}$ protocolo de pesquisa foi aprovado pela Comissão de Ética do Curso de Pós-Graduação da Faculdade de
Medicina do ABC e pelas autoridades sanitárias locais.

Os dados das adolescentes e de seus recém-nascidos foram coletados através do Sistema de Informação sobre Nascidos Vivos (SINASC), que foi implantado oficialmente no Brasil em 1990 e permite coleta individualizada, sistemática e padronizada de dados relativos à mãe e ao recém-nascido (idade, escolaridade, bairro de moradia, local em que ocorreu o nascimento, número de filhos, tipo de parto, peso ao nascer, idade gestacional do recém-nascido, sexo do recém-nascido). As informações socioeconômicas do Município de Santo André foram obtidas da Fundação Sistema Estadual de Análise de Dados (SEADE), do Instituto Brasileiro de Geografia e Estatística (IBGE) $(18,19,21-23)$ e do Mapa da exclusão/inclusão social da cidade de Santo André (13).

Foi determinada a distribuição das adolescentes nos 93 bairros de acordo com o seu local de residência. Nesse procedimento, as áreas do Mapa da exclusão/inclusão social da cidade de Santo André (13) foram compatibilizadas com as 127 áreas censitárias do Município.

O Mapa da exclusão/inclusão social da cidade de Santo André (13) foi elaborado através da construção de indicadores compostos (medidas que associam diferentes variáveis socioeconômicas em um indicador sintético de forma a permitir a análise de características de grupos populacionais que vivem em determinadas áreas geográficas) (11). Esses indicadores foram elaborados a partir de um conjunto de 14 variáveis socioeconômicas já existentes nos bancos de dados municipais. As 14 variáveis foram agrupadas em quatro dimensões, entendidas por Sposati (13) como as dimensões da exclusão/inclusão social: 1) autonomia (renda do chefe da família); 2) desenvolvimento humano (alfabetização do chefe da família, alfabetização tardia e longevidade, definida como a porcentagem da população com 70 anos ou mais); 3) qualidade de vida (domicílios servidos por água e esgoto, domicílios servidos por coleta de lixo, densidade habitacional, concentração 
de moradias precárias, domicílios não próprios, cobertura por creches para a faixa etária de 0 a 4 anos, cobertura por escola para a faixa etária de 4 a 6 anos e cobertura de um consultório de unidade básica de saúde por 5000 habitantes); e 4) eqüidade (concentração de mulheres chefes de família e concentração de mulheres chefes de família que não são alfabetizadas).

Conforme descrito anteriormente (13), a composição dessas quatro dimensões para cada um dos 93 bairros gerou seus respectivos índices de exclusão ou inclusão social. Essa composição partiu da fixação de "padrões básicos de inclusão", aos quais foi convencionado o valor 0 (zero), e da elaboração de uma escala a partir deles. A escala variou de -1 a +1 , sendo -1 considerado exclusão e +1 inclusão. $\mathrm{O}$ padrão básico de inclusão é aquele valor que indica "um mínimo de dignidade" dentro da base material real existente na cidade, ou de parâmetros já consagrados em nível internacional ou nacional, ou ainda de valores fixados como aspiração a ser atingida. Por exemplo, para a variável "renda do chefe de família" convencionou-se que $0 \%$ de chefes de família sem renda seria o padrão básico de inclusão. Os bairros com valores acima desse padrão obtiveram valores entre $+0,1 \mathrm{e}$ +1 . Os bairros que possuíam mais do que $0 \%$ de chefes de família abaixo da linha de pobreza ficaram com valores entre $-0,1$ e -1 .

Assim, os 93 bairros foram classificados em função dos índices respectivos para cada uma das 14 variáveis, para cada dimensão e para o conjunto das quatro dimensões através da soma simples dos seus índices $[-0,1+0,25+$ ..... $\left.\left(\mathrm{n}_{14}\right)\right]$. As quatro áreas homogêneas foram produzidas tomando por base quatro quartis de índices de inclusão/exclusão ( -1 a $-0,52 ;-0,51$ a $-0,02 ;-0,01$ a 0,$51 ; 0,52$ a 1$)$ e não a sua proximidade geográfica, embora tenham sido encontrados agregados homogêneos geograficamente próximos (figura 1).

Nas áreas 1 e 2, com as piores condições socioeconômicas, residia 55,3\% da população: 21,7\% na área 1 (índices de exclusão -1 a $-0,52$ ) e $33,6 \%$ na
FIGURA 1. Mapa da inclusão social do Município de Santo André (SP), Brasila ${ }^{a}$

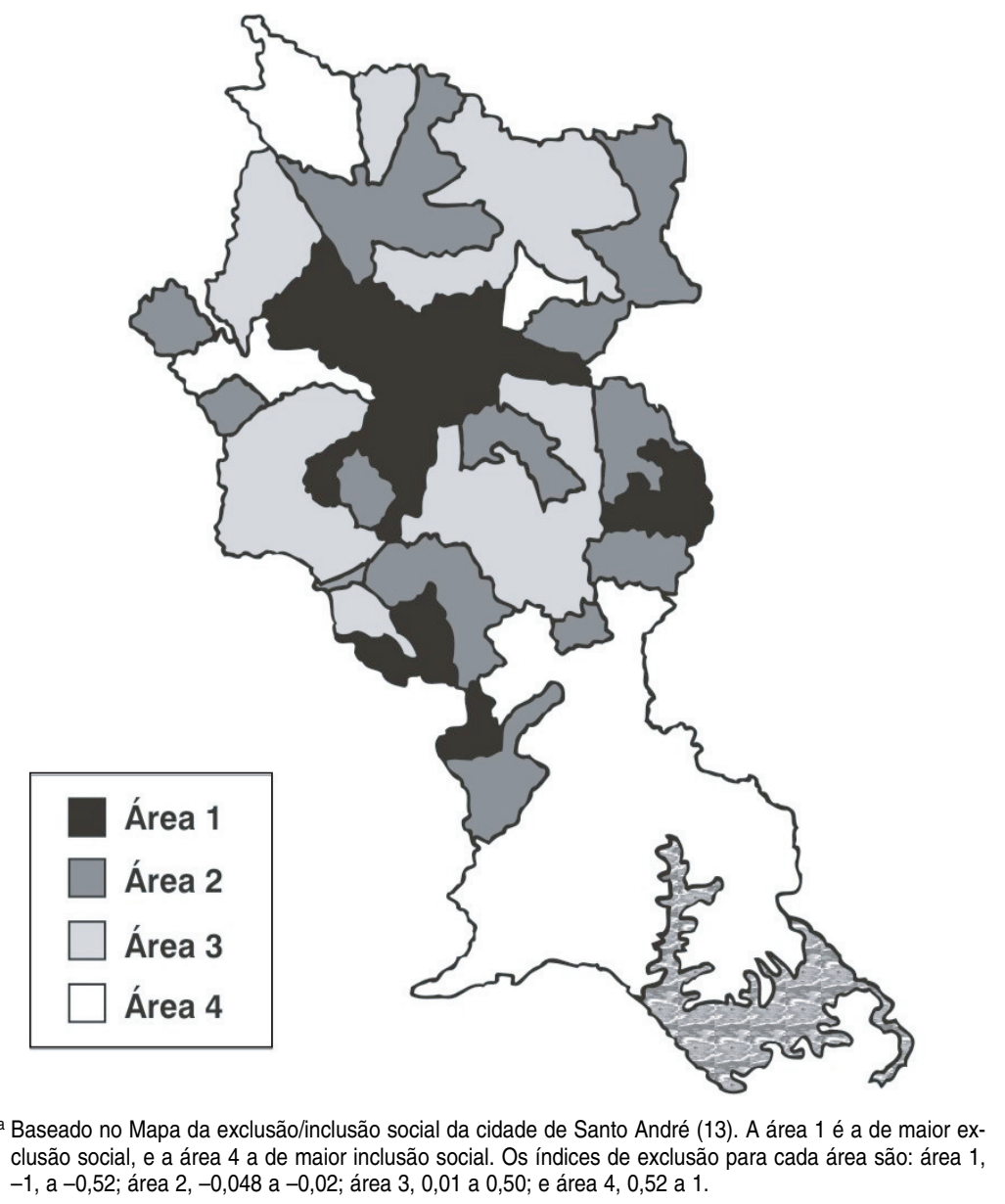

área 2 (índices de exclusão de $-0,51$ a - 0,02). A área 3, considerada como apresentando condições socioeconômicas intermediárias, abrigava 35,4\% da população (índices de inclusão de 0,01 a 0,50 ). A área 4 , que apresentava as melhores condições socioeconômicas, abrigava 9,3\% da população total do Município (índices de inclusão de $0,52$ a 1$)$.

\section{A população de adolescentes e as variáveis estudadas}

Em 1998, foram registrados 1777 nascimentos de mães adolescentes (19), o que correspondeu a $16 \%$ do total de 11043 nascidos vivos de mães de todas as idades. Foram excluídas as adolescentes que deram à luz no Município mas que ali não residiam; as que residiam em bairros não oficialmente pertencentes ao Município; e aquelas em cuja Declaração de Nascido Vivo não constava o bairro de residência. Esses critérios levaram à exclusão de 456 adolescentes. Assim, foram incluídas 1314 adolescentes e 1321 nascidos vivos (sete partos gemelares).

As seguintes variáveis contidas na Declaração de Nascido Vivo foram coletadas para a realização do estudo: 1) o hospital em que a adolescente deu à luz $(\mathrm{n}=12)$; 2) bairro de moradia; 3) idade; 4) escolaridade; 5) número de filhos; 6) número de consultas prénatais; 7) idade gestacional; 8) tipo de parto e 9) peso ao nascer.

A taxa de fecundidade específica para cada uma das áreas homogêneas foi calculada como o número de nasci- 
dos vivos no ano em cada área dividido pelo total de mulheres de 15 a 19 anos e multiplicado por 1000 (24).

No presente artigo, as quatro áreas homogêneas definidas no Mapa da exclusão/inclusão social da cidade de Santo André foram comparadas em termos do nível de escolaridade das mães adolescentes, peso ao nascer dos bebês e taxas de fecundidade.

\section{Análise estatística}

A análise dos dados foi realizada através do Programa Epi Info 6.0. Inicialmente, foi efetuada uma análise descritiva das variáveis para caracterização geral das adolescentes. Procedeuse à análise univariada para identificar as variáveis associadas às áreas homogêneas de exclusão/inclusão social. Para a análise dos diferenciais intra-urbanos de nascidos vivos e das áreas de moradia das adolescentes, foram utilizados o teste do quiquadrado $\left(\chi^{2}\right)$ e o teste de Fisher. As associações consideradas estatisticamente significativas foram aquelas que apresentaram nível de significância menor do que 5\% $(P<0,05)$.

\section{RESULTADOS}

A média de idade das adolescentes foi de 17,4 anos, sendo que a idade mínima observada foi de 12 anos $(n=2)$. Quanto maior a idade, maior o número de partos: $53,1 \%$ dos partos cor-

TABELA 1. Distribuição das adolescentes e dos nascidos vivos segundo áreas homogêneas de exclusão social, Município de Santo André (SP), Brasil, 1998

\begin{tabular}{lrlcrlr}
\hline Área $^{\mathrm{a}}$ & $\begin{array}{c}\text { Número de } \\
\text { adolescentes }(\%)\end{array}$ & $\begin{array}{c}\text { Taxa de fecundidade } \\
\text { específica }^{\mathrm{b}}\end{array}$ & $\begin{array}{c}\text { Número de nascidos } \\
\text { vivos }(\%)\end{array}$ & $\begin{array}{c}\text { Freqüência } \\
\text { acumulada em \% }\end{array}$ \\
\hline 1 & 13686 & $(23,4)$ & 35,7 & 489 & $(37,0)$ & 37,0 \\
2 & 20322 & $(34,7)$ & 25,1 & 511 & $(38,7)$ & 75,7 \\
3 & 19343 & $(33,0)$ & 13,1 & 258 & $(19,5)$ & 95,2 \\
4 & 5216 & $(8,9)$ & 12,1 & 63 & $(4,8)$ & 100,0 \\
Total & 58567 & $(100,0)$ & & 1321 & $(100,0)$ & 37,0 \\
\hline
\end{tabular}

Fonte: Sistema de Informação sobre Nascidos Vivos (SINASC), 1998.

a Conforme o Mapa da exclusão/inclusão social da cidade de Santo André (13). A área 1 é a de maior exclusão social, e a área 4 a de maior inclusão social.

${ }^{\mathrm{b}}$ Numero de nascidos vivos dividido pelo total de mulheres de 15 a 19 anos e multiplicado por 1000.

responderam à faixa de 18 a 19 anos; $37,8 \%$, à faixa de 16 a 17 anos; $8,4 \%$ à faixa de 14 a 15 anos; e $0,7 \%$ à faixa de 12 a 13 anos.

A tabela 1 mostra a distribuição de todas as 58567 adolescentes do Município nas quatro áreas homogêneas. A área 2 continha o maior número de adolescentes. Observou-se que 58,1\% moravam nas áreas 1 e 2, que têm as piores condições de vida. Também na área 2 ocorreram $75,7 \%$ dos nascimentos (tabela 1). Verificou-se uma diferença estatisticamente significativa $\left(\chi^{2}\right)$ entre a proporção de gestantes da área 4 (menor proporção) e da área 1 $(P<0,0001)$.

Ao analisar cada área em relação às demais quanto à distribuição dos nascidos vivos (teste de Fisher), verificamos que todas as áreas eram diferentes entre si, exceto a 3 e a $4(P=0,52)$. A comparação entre a área 1 e 2 foi significativa, da mesma forma como a comparação entre as áreas 1 e 3,1 e 4, 2 e 3, e 2 e 4 ( $P<0,0001$ para todas as comparações).

A tabela 1 também mostra que a taxa de fecundidade específica para cada área diminuiu na medida da inclusão social. A área 1, mais excluída, teve uma taxa de fecundidade específica de 35,7 em 1000 adolescentes, seguida da área 2, com 25,1 em 1 000, da área 3, com 13,3 em 1000 e da área 4, a menos excluída, com 12,1 em 1000.

A tabela 2 mostra o perfil de escolaridade das adolescentes e como ele se distribuiu entre as quatro áreas homogêneas. Considerando o grupo como um todo, 1160 adolescentes $(88,2 \%)$ eram alfabetizadas, $807(69,5 \%)$ possuíam ensino fundamental incompleto, $223(19,2 \%)$ tinham o ensino fundamental completo e $130(11,2 \%)$ possuíam ensino secundário (completo ou incompleto).

Ao ser analisada a escolaridade por áreas de exclusão/inclusão social,

TABELA 2. Distribuição das adolescentes segundo a escolaridade e as áreas homogêneas de exclusão social, Santo André (SP), Brasil, 1998

\begin{tabular}{|c|c|c|c|c|c|c|c|c|c|c|}
\hline \multirow[b]{3}{*}{ Escolaridade $^{b}$} & \multicolumn{8}{|c|}{ Área $^{a}$} & & \\
\hline & \multicolumn{2}{|c|}{1} & \multicolumn{2}{|c|}{2} & \multicolumn{2}{|c|}{3} & \multicolumn{2}{|c|}{4} & \multicolumn{2}{|c|}{ Total } \\
\hline & No. & $\%$ & No. & $\%$ & No. & $\%$ & No. & $\%$ & No. & $\%$ \\
\hline Nenhuma & 4 & 0,8 & 5 & 1,0 & 3 & 1,2 & 0 & 0,0 & 12 & 0,9 \\
\hline Fundamental incompleto & 346 & 71,3 & 312 & 61,1 & 120 & 47,1 & 29 & 46,0 & 807 & 61,4 \\
\hline Fundamental completo & 68 & 14,0 & 95 & 18,6 & 51 & 20,0 & 10 & 15,9 & 224 & 17,0 \\
\hline Secundário incompleto & 0 & 0,0 & 0 & 0,0 & 2 & 0,8 & 0 & 0,0 & 2 & 0,2 \\
\hline Secundário completo & 24 & 4,9 & 47 & 9,2 & 43 & 16,9 & 14 & 22,2 & 128 & 9,7 \\
\hline Sem informação & 43 & 8,9 & 52 & 10,2 & 36 & 14,1 & 10 & 15,9 & 141 & 10,7 \\
\hline Total & 485 & 100,0 & 511 & 100,0 & 255 & 100,0 & 63 & 100,0 & 1314 & 100,0 \\
\hline
\end{tabular}

Fonte: Sistema de Informação sobre Nascidos Vivos (SINASC), 1998.

${ }^{a}$ Conforme o Mapa da exclusão/inclusão social da cidade de Santo André (13). A área 1 é a de maior exclusão social, e a área 4 a de maior inclusão social. 
TABELA 3. Distribuição do baixo peso ao nascer segundo áreas homogêneas de exclusão social, Santo André (SP), Brasil, 1998

\begin{tabular}{|c|c|c|c|}
\hline Área ${ }^{a}$ & Nascidos vivos & $\begin{array}{c}\text { Nascidos vivos } \\
\text { com peso }<2500 \mathrm{~g}(\%)^{\mathrm{b}}\end{array}$ & $\begin{array}{c}\text { Baixo peso } \\
\%\end{array}$ \\
\hline 1 & 489 & $50 \quad(40,0)$ & 10,2 \\
\hline 2 & 511 & $46 \quad(36,8)$ & 9,0 \\
\hline 3 & 253 & $24 \quad(19,2)$ & 9,0 \\
\hline 4 & 63 & $5 \quad(4,0)$ & 8,0 \\
\hline Total & 1321 & $125 \quad(100,0)$ & 9,5 \\
\hline
\end{tabular}

Fonte: Sistema de Informação sobre Nascidos Vivos (SINASC), 1998.

${ }^{a}$ Conforme o Mapa da exclusão/inclusão social da cidade de Santo André (13). A área 1 é a de maior exclusão social, e a área 4 a de maior inclusão social.

${ }^{\mathrm{b}} \mathrm{A}$ porcentagem se refere ao total de nascidos vivos de baixo peso.

${ }^{c} \mathrm{~A}$ porcentagem se refere ao total de nascidos vivos em cada área.

observou-se uma relação estatisticamente significativa $(P<0,001)$, com um número maior de adolescentes de menor escolaridade nas áreas 1 e 2 em comparação com as áreas 3 e 4.

$\mathrm{O}$ registro do peso ao nascer foi encontrado em $100 \%$ das Declarações de Nascidos Vivos consultadas. O peso médio ao nascer foi de $3089 \mathrm{~g}$. Dos 1321 nascimentos analisados, o percentual de baixo peso ao nascer (BPN), ou seja, <2 $500 \mathrm{~g}$, foi de $9,5 \%$. O peso foi igual ou acima de $2500 \mathrm{~g}$ em 90,5\% os nascido vivos. Dos 125 casos de BPN, $45 \%$ eram prematuros. Foram encontrados 33 nascidos vivos com peso maior do que $4000 \mathrm{~g}(2,5 \%)$.

A duração da gestação foi analisada no intuito de se observar a taxa de prematuridade e pós-datismo nas adolescentes. Os intervalos considerados no presente estudo são os mesmos utilizados na Declaração de Nascido Vivo.

Em relação à distribuição dos nascidos vivos segundo a idade gestacional, observou-se que 1233 (93,3\%) tinham de 37 a 41 semanas de idade; 73 (5,5\%) tinham idade gestacional menor do que 36 semanas e foram considerados prematuros. Houve um caso de mais de 42 semanas. Em duas Declarações de Nascido Vivo a informação foi dada como ignorada e em $12(0,2 \%)$ o registro estava em branco. Quando analisada a distribuição das adolescentes segundo a idade gestacional e as áreas de exclusão social, observou-se que não houve diferença entre as áreas $(P=0,81)$.

A tabela 3 analisa a distribuição do BPN entre as quatro áreas de estudo. A maioria dos bebês com BPN $(76,8 \%)$ encontravam-se nas áreas 1 e 2, as mais pobres.

\section{DISCUSSÃO}

A proporção de $16 \%$ de nascidos vivos de mães adolescentes encontrada neste estudo é menor do que a encontrada para o Brasil no mesmo ano, 23,2\% (25). Almeida (26), estudando 3225 nascidos vivos de mães residentes em Santo André em 1992, encontrou $15,5 \%$ de mães adolescentes.

Mello Jorge et al. (27) encontraram, nos municípios em que a principal atividade econômica era a agropecuária, uma proporção de nascidos vivos em adolescentes de em torno de $29,1 \%$, enquanto que, em São José dos Campos, município com características industriais semelhantes a Santo André, essa taxa foi de $15 \%$.

Diversos autores afirmam que os padrões sexuais e reprodutivos variam de acordo com as regiões, e que essas diferenças se devem a inúmeros fatores: caráter rural ou urbano da região, condições socioeconômicas, diferenças culturais e acesso a serviços de saúde e a métodos anticoncepcionais, determinando maior ou menor incidência de gravidez na população adolescente. Além disso, culturas regionais, estilos de vida, projetos de vida, opções de futuro, perspectivas de estudo e casamento, enfim, um leque de possibilidades que variam de região para região, determinam as variações nas taxas das diversas localidades $(2,5,28)$. Guzmán et al. (29) indicam uma diminuição na taxa de fecundidade geral nas Américas no momento atual, sendo que, segundo Gómez (30), a gravidez na adolescência na América Latina atinge 20\% dos nascidos vivos. No Brasil, observase um aumento na proporção de nascidos vivos em mães adolescentes ao longo dos últimos anos em relação às demais idades maternas. Em 1980 essa proporção foi $12,8 \%$, contra $13,8 \%$ em 1985, 16\% em 1990, 18,7\% em 1995 e 23,2\% em 1998 (18, 21-23). Campanário e Yazaki (31) e Martins e Almeida (32) concordam que a fecundidade no Brasil tem apresentado uma queda nas últimas décadas; no entanto, Martins e Almeida (32) demonstram que, quando analisada a fecundidade e a idade da mulher, o grupo de adolescentes teve significativa participação na fecundidade total.

Quando comparados os diversos países, observa-se que a taxa de fecundidade específica varia. Guzmán et al. (29), estudando a fecundidade na América Latina, observaram maiores taxas na Nicarágua (157,2 em 1000 adolescentes), Guatemala (126,4 em 1 000), El Salvador (123 em 1 000), Venezuela (101,4 em 1 000), Paraguai (95 em 1 000), Bolívia (94 em 1 000), Colômbia (92 em 1 000), República Dominicana (91,2 em 1 000), Brasil (82,4 em $1000)$ e Costa Rica (89 em 1000 ), sendo as taxas mais baixas as do Uruguai (70,5 em 1 000), Argentina (69,7 em $1000)$ e Chile (67,3 em 1000$)$.

Na República dos Camarões, Locoh (33) observou uma taxa de 165 em 1000 adolescentes. Nos países desenvolvidos, Singh e Darroch (34) observaram situação totalmente distinta. Holanda, Itália e Espanha apresentaram em seus estudos uma taxa de fecundidade específica de 12 em 1 000, igual à encontrada neste estudo para as adolescentes das áreas mais ricas. Canadá, Austrália e países do Oeste da Europa apresentaram taxas entre 20 e 50 em 1000 . O estudo apontou, no entanto, que alguns países desenvolvidos, principalmente os Estados Unidos, apresentaram taxas superiores a 50 em 1000.

Os resultados deste estudo permitiram concluir que a taxa de fecundi- 
dade específica do Município de Santo André foi de 60 em 1000 adolescentes, menor do que a do Brasil e semelhante à do Chile e à dos Estados Unidos.

Quanto à distribuição da taxa de fecundidade específica, os resultados de nosso estudo são comparáveis aos de Martins e Almeida (32), que analisaram a fecundidade no Município de São Paulo, também através de diferenciais intra-urbanos. Aqueles autores encontraram diferenças entre as áreas de maior índice de desenvolvimento humano (IDH), que apresentaram uma taxa de fecundidade específica de 38,7 em 1 000, comparada com 89,6 em 1000 para áreas com menor IDH.

A análise da gravidez na adolescência através da diferença entre os bairros em uma cidade permite localizar e dimensionar uma das situações de saúde que mais preocupa os órgãos públicos e profissionais de saúde, assim como dimensionar recursos propriamente ligados à saúde, número de postos de saúde, número de leitos, e programas de saúde para essa população específica. Essa análise permite também efetivar o incentivo a promoção e prevenção da gravidez na adolescência, que envolve recursos sociais, econômicos e culturais que extrapolam a questão de saúde, como escolas, lazer, esportes, além das condições de vida básicas, como rede de água e esgoto, moradia e número de pessoas por domicílio, dentre outras. O conhecimento dessas realidades permitirá traçar políticas públicas para a população adolescente em geral, com ênfase na área de saúde reprodutiva, visando à saúde integral da adolescente e de seu filho.

Certos autores $(6,29,35,36)$ sugerem uma importante associação entre os níveis de pobreza e a fecundidade, demonstrando que é entre as adolescentes mais pobres que o número de partos é maior. A literatura aponta ainda que o grau de instrução é um fator de exposição ao risco de gravidez não planejada no grupo de adolescentes (37-39). As estatísticas mostram que as adolescentes que ficam grávidas têm menor grau de instrução, ou que já estavam fora da escola no momento da gravidez $(40,41)$.
Para certos autores, as adolescentes grávidas de baixa escolaridade estariam cumprindo, de certa forma, o seu papel social possível, com as limitações que o mundo moderno impõe a quem não tem estudo. Para certas populações, a gravidez precoce funciona como fator de exclusão social, levando a menores possibilidades de melhorar as condições de vida. Nessa lógica, as mulheres que têm mais tempo de educação formal têm maior probabilidade de adiar a procriação e o casamento do que aquelas com pouca ou nenhuma instrução. As mulheres que começam a ter filhos cedo raramente voltam à escola (38).

A Organização Pan-Americana de Saúde (OPAS) afirma que a gravidez na adolescência é uma porta de entrada para a pobreza, pois leva a uma diminuição do leque de possibilidades sociais e econômicas, inclusive em termos do acesso à escola (42). A exigência do mundo moderno, industrializado e informatizado, não absorveria essa mão-de-obra desqualificada e não preparada para as exigências do mercado de trabalho, perpetuando a situação de pobreza para a jovem e o seu filho. Os estudos afirmam ainda que a mulher que inicia a sua vida reprodutiva antes dos 20 anos tem menores chances de se emancipar socialmente $(42,43)$. Dessa forma, o fator escolaridade demonstra ser um indicador socioeconômico importante, e muitas vezes determinante, para o futuro da adolescente e de seu filho.

O BPN é apontado por alguns autores como um indicador de desenvolvimento social, pois reflete a interação entre fatores sociais, econômicos, demográficos e de atenção à saúde, sendo inclusive considerado mais importante do que indicadores econômicos como o produto interno bruto per capita (44). Como apontam alguns estudos (45-47), o peso ao nascer é reconhecido pela Organização Mundial da Saúde (OMS) como o determinante isolado mais importante da sobrevivência infantil, que atua como importante fator de risco para a mortalidade. O presente estudo encontrou 9,5\% de BPN. Também no Brasil, Almeida (26) encontrou a proporção de $6,8 \%$ entre todas as idades maternas, e Mello Jorge et al. (27) en- contraram 7,6\%. Galletta (48), estudando 100 gestantes adolescentes submetidas ao pré-natal na Universidade de São Paulo (USP) entre 1992 e 1994, encontrou uma incidência de baixo peso de $14,8 \%$. Esse autor aponta o crescimento intra-uterino retardado como o fator mais provável para explicar a alta incidência observada pelo estudo.

Costa (47) encontrou, em Juiz de Fora, Estado de Minas Gerais, 11,4\% de BPN. Segundo o autor, a OMS considera satisfatório um percentual em torno de $6 \%$. Pinto e Silva (37) encontraram 21,1\% para o Brasil, Chemello (49) encontraram $15,3 \%$ no Rio Grande do Sul, Cruz (50) encontrou 18,4\% de BPN em uma maternidade em São Paulo, e Fabrício et al. (51) encontraram 10,3\% na Santa Casa de Misericórdia de São Paulo.

$O$ peso foi informado em todas as 1321 Declarações de Nascido Vivo analisadas por este estudo, o que facilitou a compreensão do nível de saúde dos recém-nascidos e de suas possibilidades de sobrevivência. A taxa de BPN encontrada, de 9,5\%, embora longe da preconizada pela OMS, foi considerada baixa se comparada aos demais estudos, visto que foi avaliado todo o Município de Santo André, ou seja, diversos grupos sociais e realidades econômicas.

A distribuição dos nascidos vivos segundo a idade gestacional demonstrou que a proporção de prematuros foi de 5,5\%, um total de 73 casos. Pinto e Silva (37) aponta que a idade tem sido relacionada com a prematuridade nos extremos da vida reprodutiva, mas salienta que a metodologia utilizada pelos estudos e os dados colhidos através das Declarações de Nascido Vivo têm limitações. O autor entende que o melhor método seria a utilização do método de Capurro, e chama a atenção quanto à enorme variabilidade na incidência de prematuridade, não só entre adolescentes, como na população em geral, que varia de 7 a $37,8 \%$.

\section{CONCLUSÕES}

No presente estudo, as áreas 1 e 2, de maior exclusão social, continham o 
maior número de nascidos vivos de adolescentes. Os resultados mostram uma diferença estatisticamente significativa entre as áreas de exclusão social, ocorrendo diminuição na proporção de nascidos vivos da área 1 para a área 4, a mais favorecida. As maiores taxas de fecundidade específica foram observadas para as áreas com piores condições socioeconômicas. As áreas mais pobres também concentraram o maior número de adolescentes com menor nível de escolaridade e de bebês com BPN.

Devido às diferenças importantes encontradas no Município de Santo André, caberia sugerir a formulação de políticas públicas com uma visão intersetorial integrada, ou seja, com foco na saúde integral do grupo adolescente, inclusive na educação, esporte, lazer e cultura. Dentro do princípio de promoção à saúde, é necessário investir na expansão de capacidades das adolescentes para que se fortaleça a sua autonomia e para que possam fazer escolhas saudáveis, compatíveis com o seu projeto de vida. Esse trabalho com sujeitos sociais não pode existir sem a instituição de um modelo de prioridades sociais no qual a lógica econômica não prevaleça sobre a melhoria das condições de vida, a cooperação e a solidariedade.

\section{REFERÊNCIAS}

1. Brasil, Ministério da Saúde. PROSAD-Programa de saúde do adolescente: bases programáticas. Brasilia: Ministério da Saúde; 1989.

2. Organización Pan-Americana de la Salud. Recomendaciones para la atención integral de salud de los adolescentes con énfasis en salud sexual y reproductiva. Washington, D.C.: OPS; 2000. (Serie OPS/FNUAP, no. 2).

3. The Allan Guttmacher Institute. Into a new world: young women's sexual and reproductive lives. New York: The Allan Guttmacher Institute; 1998.

4. Federação Brasileira das Sociedades de Ginecologia e Obstetrícia. Gravidez na adolescência: uma questão de saúde pública. J FEBRASGO. 2000;7(3):7-10.

5. Ferraz EA, Ferreira IQ. Início da atividade sexual e características da população adolescente que engravida. Em: Vieira E. Seminário de gravidez na adolescência. Rio de Janeiro: Ministério da Saúde; 1998. Pp. 47-56.

6. Maddaleno M. Prefacio. Em: Organización Pan-Americana de la Salud. Recomendaciones para la atención integral de salud de los adolescentes con énfasis en salud sexual y reproductiva. Washington, D.C.: OPS; 2000. (Serie OPS/FNUAP, no. 2). Pp. 3-5.

7. Akerman M, Bousquat A. Mapas de risco de violência. São Paulo Perspec. 1999;13(4):112-20.

8. Akerman M, Campanário P, Maia PB. Saúde e meio ambiente: análise de diferenciais intraurbanos, Município de São Paulo, Brasil. Rev Saude Publica. 1996;30(4):372-82.

9. Medronho R. Geoprocessamento e saúde: uma nova abordagem do espaço no processo saúde-doença [dissertação]. Rio de Janeiro: Fundação Oswaldo Cruz; 1995.

10. Castellanos PL. Epidemiologia, saúde pública, situação de saúde e condições de vida: considerações conceituais. Em: Barata RB. Condições de vida e situação de saúde. Rio de Janeiro: ABRASCO; 1997. Pp. 31-75.

11. Akerman M. A construção de indicadores compostos para projetos de cidades saudáveis: um convite ao pacto transetorial. Em: Mendes $\mathrm{EV}$, org. A organização de saúde no nível local. São Paulo: Hucitec; 1998. Pp. 319-35.

12. Berlinguer G. Ética da saúde. $2^{a}$ ed. São Paulo: Hucitec; 1996.

13. Sposati A. Mapa da exclusão/inclusão social da cidade de Santo André. Santo André: Pontifícia Universidade Católica de São Paulo;
2000. Disponível em: http://www.dpi.inpe. br/geopro/exclusao/oficinas/mapa2000.pdf. Acessado em janeiro de 2006.

14. Mandanipour A, Cars G, Allen J. Social exclusion in European cities. London: Jessica Kingsley; 1998.

15. Byme D. Social exclusion. Buckingham: Open University Press; 1999.

16. Bonnell CP, Strange VJ, Stephenson JM, Oakley AR, Copas AJ, Forrest SP, et al. Effect of social exclusion on the risk of teenage pregnancy: development of hypotheses using baseline data from a randomized trial of sex education. J Epidemiol Community Health. 2003;57:871-6.

17. Garrafa V. Saúde pública, bioética e eqüidade. Em: Anais do Congresso Brasileiro de Saúde Coletiva. Águas de Lindóia, SP, 1997. Rio de Janeiro: Abrasco; 1997. Pp. 59-67.

18. Instituto Brasileiro de Geografia e Estatística. Anuário estatístico 1997. Rio de Janeiro: IBGE; 1998.

19. Fundação Sistema Estadual de Análise de Dados. Anuário estatístico do Estado de São Paulo. São Paulo: SEADE; 1998.

20. Organização Mundial de Saúde. A saúde dos jovens. Em: Assembléia Mundial de Saúde. Genebra: OMS; 1989. (Relatório técnico).

21. Instituto Brasileiro de Geografia e Estatística. Anuário estatístico 1980. Rio de Janeiro: IBGE; 1981.

22. Instituto Brasileiro de Geografia e Estatística. Anuário estatístico 1985. Rio de Janeiro: IBGE; 1986.

23. Instituto Brasileiro de Geografia e Estatística. Anuário estatístico 1990. Rio de Janeiro: IBGE; 1991.

24. Rouquayrol MZ, Pontes KL. A medida da saúde coletiva. Em: Rouquayrol MZ, Almeida Filho N. Epidemiologia e saúde. $5^{\mathrm{a}}$ ed. Rio de Janeiro: Medsi; 1999. Pp. 64-8.

25. Ministério da Saúde, DATASUS. Gravidez na adolescência: atendimento pelo sistema único de saúde, 1998. Disponível em: http://w3. datasus.gov.br/datasus/datasus.php. Acessado em janeiro de 2006.

26. Almeida MF. Mortalidade neonatal em Santo André [tese]. São Paulo: Universidade de São Paulo, Faculdade de Saúde Pública; 1994.

27. Mello Jorge MHP, Gotileb SLD, Soboll MLMS, Almeida MF, Latorre MRDO. Avaliação do sistema de informação sobre os nascidos vivos e o uso de seus dados em epidemiologia e estatísticas de saúde. Rev Saude Publica. 1993;27(supl):1-46.

28. Coll A. Embarazo en la adolescencia? Cuál es el problema? Em: Burak SD. Adolescencia y juventud en América Latina. Costa Rica: LUR; 2001. Pp. 425-45.

29. Guzmán JM, Contreras JM, Hakkert R. La situación actual del embarazo y el aborto en la adolescencia en América Latina y el Caribe. Em: Burak SD. Adolescencia y juventud en América Latina. Costa Rica: LUR; 2001. Pp. 391-424.

30. Gómez JRL. El embarazo de la adolescente. Em: Gómez JRL, López CB. Salud del adolescente. Valencia, Venezuela: Clemente Editores CA; 1997. Pp. 161-71.

31. Campanário P, Yazaki LMA. Fecundidade em São Paulo e suas regiões de governo: níveis e tendências. Em: Fundação Sistema Estadual de Análise de Dados. A fecundidade da mulher paulista. São Paulo: SEADE; 1994. Pp. $12-$ 76. (Informe demográfico $\mathrm{n}^{\circ} 25$ ).

32. Martins CM, Almeida MF. Fecundidade e diferenciais intra-urbanos de desenvolvimento humano, São Paulo, Brasil, 1997. Rev Saude Publica. 2001;35(5):421-7.

33. Locoh T. [Premature fertility in Sub-Saharan Africa].Chron CEPED. 1994:Summer;(14):1-3.

34. Singh S, Darroch JE. Adolescent pregnancy and childbearing: levels and trends in developed countries. Fam Plann Perspect. 2000; 32(1):14-23.

35. Stern C. El embarazo en la adolescencia como problema público: una visión crítica. Salud Publica Mex. 1997;39(2):137-43.

36. Monteiro DLM. Modelo de assistência multidisciplinar na adolescência visando o bemestar materno e fetal e a contracepção. Reprodução e Climatério. 1995;10(2):67-72.

37. Pinto e Silva JL, Motta ML. Gravidez entre adolescentes muito jovens. Femina. 1994; 22(5):348-54.

38. Population reports. Como satisfazer as necessidades de adultos jovens. Baltimore: The Johns Hopkins University; 1995. [Série J(41)].

39. Bastos AC. Contracepção. Em: Bastos AC. Ginecologia infanto-juvenil. $3^{\text {a }}$ ed. São Paulo: ROCA; 1998. Pp. 173-9.

40. Castello Branco VM. Gravidez na adolescência: problema para quem? Rev NATES (Juiz de Fora). 2000;5(3):44. 
41. Maia Filho NL. Gravidez entre adolescentes precoces: um evitável problema social. J Bras Ginecol. 1994;104(10):363-7.

42. Organización Mundial de la Salud. La salud de los adolescentes y jovenes en las Américas: un compromiso con el futuro. Washington, D.C.: OPS; 1985.

43. Population reports. A juventude na década de 1980: problemas sociais e de saúde. Baltimore: The Johns Hopkins University; 1987. [Série $\mathrm{M}(9) 1-44]$.

44. Barros FC, Victora CG, Teixeira AMB, Puerto Filho M. Mortalidade perinatal e infantil em Pelotas, RS: nossas estatísticas são confiáveis? Cad Saude Publica. 1985;1(3):348-58.

45. Laurenti R, Buchalla CM. Mortalidade perinatal segundo peso ao nascer, idade materna, assistência pré-natal e hábito de fumar da mãe. Rev Saude Publica. 1985;19(3):225-32.
46. Barros FC, Victora CG. Epidemiologia da saúde infantil—um manual para diagnósticos comunitários. $3^{\mathrm{a}}$ ed. São Paulo: Hucitec/ Unicef; 1998

47. Costa TJNM. Sistema de informação sobre os nascidos vivos: uma valiosa contribuição na construção de diagnóstico de saúde. Rev NATES (Juiz de Fora). 2000;5(3):26-32.

48. Galletta MA, Lippi ATA, Giribola A, Mígueles J, Zugaib M. Resultados obstétricos e perinatais em gestantes adolescentes atendidas em pré-natal especializado. Rev Ginecol Obstet. 1997;8(1):10-9.

49. Chemello CS. Perfil epidemiológico das adolescentes grávidas na cidade de São Marcos, RS. 1999 [dissertação]. São Paulo: , Universidade de São Paulo, Faculdade de Saúde Pública; 1999.
50. Cruz AM. Gravidez na adolescência: estudo das condições do recém-nascido de baixo peso de mães adolescentes atendidas no Hospital Municipal e Maternidade Escola Dr. Mário de Moraes Altenfelder Silva [dissertação]. São Paulo: Universidade de São Paulo, Faculdade de Saúde Pública; 1997.

51. Fabrício VC. A gravidez na adolescência como fator de risco para o recém-nascido. Em: Sociedade Brasileira de Pediatria. Congresso Brasileiro de Adolescência. Gramado: SBP; 1998. P. 53.

Manuscrito recebido em 9 de setembro de 2005. Aceito em versão revisada em 20 de dezembro de 2005.

ABSTRACT Objective. To compare adolescent mothers living in four areas with different degrees of social exclusion in the city of Santo André, São Paulo, Brazil, in terms of the mothers' schooling, the birth weight and gestational age of their babies, and the specific fertility rate of each of the four areas in 1998.

Adolescent pregnancy and social exclusion: analysis of intra-urban disparities

Method. An ecological cross-sectional study was carried out with 1314 adolescent girls. The four areas analyzed had earlier been defined in the City of Santo André Social Exclusion/Inclusion Map. Area 1 had the highest exclusion index (worst socioeconomic conditions), and Area 4 the lowest exclusion index (best socioeconomic conditions). The data relating to the adolescent mothers and their children were collected from the National Live Birth Information System, and the socioeconomic data for Santo André were obtained from the State Data Analysis System Foundation, the Brazilian Institute of Geography and Statistics, and the city's Social Exclusion/ Inclusion Map.

Results. Having little formal education was statistically associated with the poorest areas. Of the infants with a birthweight $<2500 \mathrm{~g}, 76.8 \%$ of them were born in the two poorest areas of the city. The highest fertility rate (35.7 per 1000 adolescents) was found in Area 1, the area with the worst socioeconomic conditions; the lowest fertility rate (12.1 per 1000$)$ was found in Area 4, the area with the best socioeconomic conditions. The proportion of births that were premature did not differ among the four areas $(P=0.81)$.

Conclusions. The results showed that adolescent girls with little schooling and a lower socioeconomic level were more likely to give birth. Specific actions should be promoted to prevent pregnancy in this group and to foster the social inclusion of these adolescents and their children, providing them with opportunities to improve their socioeconomic situation.

Key words Social class, residence characteristics, pregnancy in adolescence, Brazil. 